\title{
1 Strong neutral sweeps occurring during a population contraction
}

2

10 Abstract

11 A strong reduction in diversity around a specific locus is often interpreted as a recent rapid

12 fixation of a positively selected allele, a phenomenon called a selective sweep. Rapid fixation of

13 neutral variants can however lead to similar reduction in local diversity, especially when the

14 population experiences changes in population size, e.g., bottlenecks or range expansions. The

15 fact that demographic processes can lead to signals of nucleotide diversity very similar to

16 signals of selective sweeps is at the core of an ongoing discussion about the roles of

17 demography and natural selection in shaping patterns of neutral variation. Here we

18 quantitatively investigate the shape of such neutral valleys of diversity under a simple model of

19 a single population size change, and we compare it to signals of a selective sweep. We

20 analytically describe the expected shape of such "neutral sweeps" and show that selective

21 sweep valleys of diversity are, for the same fixation time, wider than neutral valleys. On the

22 other hand, it is always possible to parametrize our model to find a neutral valley that has the

23 same width as a given selected valley. We apply our framework to the case of a putative

24 selective sweep signal around the gene Quetzalcoatl in D. melanogaster and show that the

25 valley of diversity in the vicinity of this gene is compatible with a short bottleneck scenario

26 without selection. Our findings provide further insight in how simple demographic models can

27 create valleys of genetic diversity that may falsely be attributed to positive selection. 


\section{Introduction}

29 Past demography and natural selection play a critical role in shaping extant genetic diversity. A

30 central question in population genetics is to quantify their respective impact on observed

31 genomic diversity. Because selection interferes with demographic estimates and vice versa,

32 estimation of one of these two components is difficult without accounting for the other

33 (Charlesworth et al. 1993, 1995; Kaiser and Charlesworth 2009; O’Fallon et al. 2010;

34 Charlesworth 2013; Nicolaisen and Desai 2013; Johri et al. 2020, 2021b). Moreover, the relative

35 importance of demography and selection as determinants of genome wide diversity is currently

36 hotly debated, and may vary extensively among species (Corbett-Detig et al. 2015; Rousselle et

37 al. 2018; Pouyet and Gilbert 2019; Galtier and Rousselle 2020). It has been shown that selection

38 and demography can leave very similar footprints on the genetic diversity of a population

39 (Andolfatto and Przeworski 2000; Teshima et al. 2006; Thornton and Jensen 2007; Johri et al.

40 2021a). Disentangling the effects of demography and selection is therefore crucial to avoid

41 erroneous inference of evolutionary scenarios from genomic data (Jensen et al. 2005; Wares

42 2009; Mathew and Jensen 2015; Johri et al. 2020).

43 Hard selective sweeps lead to valleys of strongly reduced diversity around positively selected

44 sites due to the hitchhiking of linked neutral loci (Maynard Smith and Haigh 1974), such

45 observations of strong depletions of diversity in some genomic regions are often interpreted as

46 due to past episode of positive selection, because the probability to observe a fast fixation of a

47 neutral variant in a population of constant size is extremely low. However, during a range

48 expansion for instance, some neutral or even mildly deleterious mutations can go quickly to

49 fixation due to the low effective size of populations on the front of the range (Edmonds et al.

50 2004; Klopfstein et al. 2006; Hallatschek and Nelson 2008; Peischl et al. 2013), a phenomenon

51 termed allele surfing (Klopfstein et al. 2006). Theoretical studies have shown that the average

52 neutral diversity on the wave front decays exponentially as the range expands (Hallatschek and

53 Nelson 2008), similarly to what happens when a population experiences a sudden decay of the

54 population size, i.e. a population contraction, due to a drastic change in the environment for

55 example. In both cases, a mutation appearing when the population size is shrinking might go

56 quickly to fixation, inducing a strong decrease of diversity in the surrounding genomic region, 
57 whereas the average level of diversity might stay quite high depending on the strength and the duration of the contraction. As a result, the coalescent tree of alleles sampled in a population with strongly reduced effective population size will have short external branches, and long internal branches, depending on the parameters of the model (Excoffier et al. 2009). The site frequency spectrum associated to such a tree resembles a neutral SFS, but with a lack of rare alleles and an excess of high frequency sites, i.e. it becomes "flatter" (Sousa et al. 2014; Peischl and Excoffier 2015). The footprint left by the rapid fixation of a neutral allele on the surrounding genomic diversity, might thus be like that of a positively selected allele sweeping through a constant size population.

The expected shape of nucleotide diversity in genomic regions surrounding a site undergoing a rapid neutral fixation has been investigated analytically and numerically. Tajima (1990) studied the reduction of diversity during a neutral fixation at a given recombination distance from the fixing site. His results rely on rigorous mathematical arguments based on diffusion theory, but no closed form solution is provided for the shape of a neutral sweep. Johri et al. (2021a) described the valley of diversity occurring around a neutral fixation using an approach

72 introduced for selective sweeps, assuming that the evolution of the allele frequency is that of a 73 selected allele except in the initial stochastic phase. Here, we extend this work by inferring the dynamics of fixation of neutral alleles after a population contraction and we examine their effects on neighboring regions of the genome. We provide an analytical result for the expected coalescence time as a function of the recombination distance from the locus undergoing a fast fixation. Importantly, our results apply regardless of the process driving the allele going to

78 fixation (neutrality, positive selection, background selection), as it only relies on the typical

79 trajectory of an allele going to fixation in a given time, even though this trajectory differs

80 depending on the underlying driver of this fixation (i.e., neutrality or selection). We compare

81 our results against simulations and find that they hold for a wide range of realistic parameter

82 combinations. We compare our results about the signature of neutral sweeps to patterns

83 expected under selective sweeps and discuss potential differences between the signatures that

84 could potentially allow us to discriminate between neutral and selective processes for a given

85 demographic scenario. Finally, we investigate the similarity between the genomic signature of 
an allele going to fixation either selectively or neutrally and observe that a selective sweep signal can in principle be replicated in a neutral model with an appropriate choice of demographic parameters. To illustrate this point, we examine a classical example of a selective sweep found in the genome of D. melanogaster around the Qtzl gene (Rogers et al. 2010). We conclude that strong diversity depletions in the genome of a population, often attributed to the

91 effect of positive selection, can be obtained with demographic effects only, and we call for

92 caution when trying to detect signals of adaptation from genomic data, adding support to

93 previous studies reaching similar conclusions (Thornton and Jensen 2007; Crisci et al. 2013;

94 Jensen et al. 2019).

\section{Model}

96 We model here the effect of an instantaneous population contraction on genomic diversity.

97 Throughout the whole manuscript, time is measured backwards. We assume that $t_{\mathrm{c}}$ generations

98 before the present, the population size instantaneously dropped from $N_{0}$ diploid individuals to

$99 \quad N_{c}$ individuals with $N_{c}<N_{0}$. We assume a standard coalescent model (Kingman 1982a; b) with

100 discrete non-overlapping generations, random mating, monoecious individuals, and no

101 selection. Two haplotypes sampled in the current population at time $t=0$ have, as we go

102 backwards in time, a constant probability $\left(2 N_{c}\right)^{-1}$ of coalescing at each generation, for the first $t_{c}$

103 generations, and then this probability switches to $\left(2 N_{0}\right)^{-1}$ as we enter the ancestral

104 uncontracted population. We can approximate the distribution of coalescence time $T$ of these

105 two haplotypes as a piecewise exponential distribution (see Appendix) with expected value:

$$
\mathrm{E}[T]=2\left(N_{0}-N_{c}\right) e^{-t_{\mathrm{c}} / 2 N_{c}}+2 N_{c} .
$$

107 We see that the expected coalescence time decreases exponentially with the age of the 108 contraction $t_{c}$ and that it approaches $2 N_{c}$ for a very old contraction. Coalescence times cannot

109 be measured directly from empirical data, but they are closely related to nucleotide diversity $\pi$.

110 Under the infinitely many sites model, the number of nucleotide differences between two

111 homologous DNA segments is proportional to their coalescence time $T$ as $\pi=2 \mu T$, where $\mu$ is

112 the total mutation rate for the whole segment. Multiplying eq. (1) by $2 \mu$ shows that an 
113 instantaneous population contraction leads to an exponential decrease of the expected

114 nucleotide diversity along the genome with the age of the contraction $t_{c}$. However, it does not

115 inform us on the distribution of nucleotide diversity $\pi$ along the genome, or on spatially

116 correlated patterns of diversity such as local depletion or excess of diversity relative to the

117 expectation.
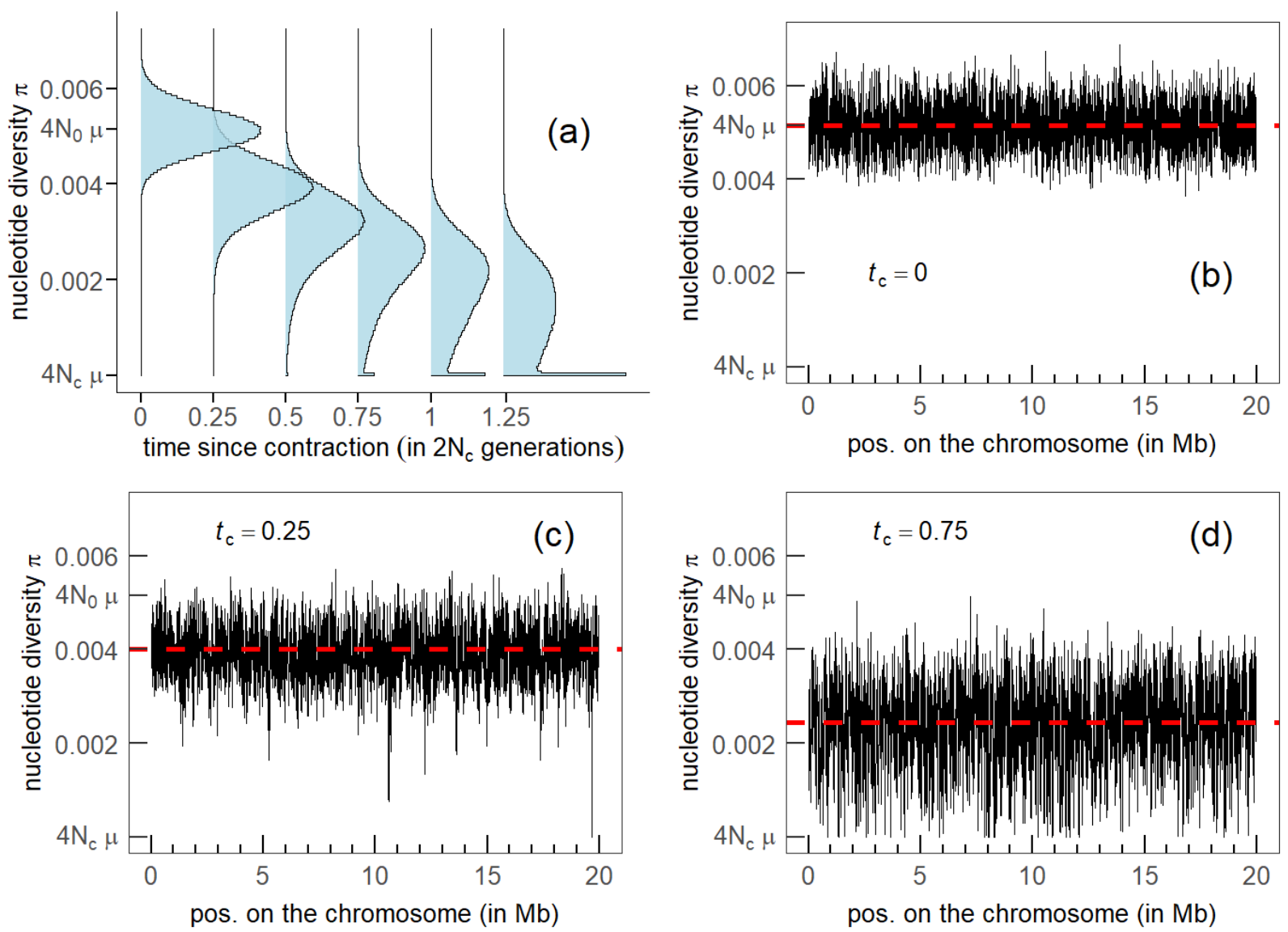

119 Figure 1. Nucleotide diversity of a population experiencing a contraction, as a function of the time $t_{c}$ elapsed since the contraction, measured in units of $2 N_{c}$. (a) distribution of nucleotide diversity as a function of time, nucleotide diversity along the chromosome at $t_{c}=0$ (panel b), at $t_{c}=0.25$ (panel c) and at $t_{c}=0.75$ (panel d). Population size before contraction $N_{0}=2.37 \times 10^{6}$ and after contraction $N_{c}=4,400$. Mutation rate $\mu=5.42 \times 10^{-10}$ per site per generation.

124 Recombination rate $r=3.5 \times 10^{-8}$ per site per generation. Chromosome size $L=20 \mathrm{Mb}$. Window size $10 \mathrm{~Kb}$ sliding at $1 \mathrm{~Kb}$ intervals. Sample size: 30 haplotypes. These parameters are taken from Rogers et al. (2010). Simulations were performed with fastsimcoal2 (Excofffier et al. 2021). 
128 Fig. 1 shows the evolution of the distribution of $\pi$ as a function of the time $t_{c}$ elapsed since the contraction. For $t_{\mathrm{c}}=0$, there is no contraction, and the population size remains constant and

130 equal to $N_{0}$. In this case we see (fig. $1 a, 1 b, t_{c}=0$ ) that the distribution of $\pi$ is symmetric and

131 centered at $\mathrm{E}[\pi]=4 N_{0} \mu$. For an older contraction, we see that the distribution is not only

132 shifted to lower values of diversity as expected from eq. (1), but that it also becomes strongly

133 peaked around $\pi=4 N_{c} \mu$. This bimodality of the distribution can be understood intuitively in the

134 following way. There are two possible types of coalescent trees for haplotypes sampled after

135 the population contraction (note that the tree depends on the locus considered because of

136 recombination). Indeed, the most recent common ancestor (MRCA) of the sample lived either

137 before the contraction $\left(T_{\mathrm{MRCA}}>t_{c}\right)$, or after the contraction $\left(T_{\mathrm{MRCA}}<t_{c}\right)$. In the former case, the

138 tree at this locus has long inner branches and short outer branches, whereas in the latter case,

139 the tree is essentially a (short) neutral tree corresponding to a population of constant size $N_{\mathrm{c}}$

140 (Excoffier et al. 2009). Both types of trees occur at different loci and correspond to the two

141 observed modes in the distribution of the nucleotide diversity along the chromosome. The

142 precise shape of the distribution of nucleotide diversity across sites depends on the relative

143 frequency of both types of trees, which itself depends on the age of the contraction $t_{c}$. For a

144 sample of size two, the probability that the MRCA lived after the contraction, that is, $T_{\text {MRCA }}<t_{c}$

145 is $1-e^{-t_{c} / 2 N_{c}}$. For a larger sample of haplotypes, there is no closed form solution for this

146 probability, but the trees rooted after the contraction are rare for $t_{c} \ll<2 N_{c}$ and very frequent

147 when $t_{c} \gg 2 N_{c}$ (Tavaré 1984). Therefore, the evolution of the distribution of $\pi$ for increasing

148 contraction age $t_{c}$ appears to be a transition from a unimodal distribution centered at $4 N_{0} \mu$ to

149 another unimodal distribution centered at $4 N_{c} \mu$, with both modes coexisting for intermediate

150 ages (fig. 1). This bimodality has been pointed out previously in the context of population

151 bottlenecks (Austerlitz et al. 1997); however, those studies mainly focused on long duration

152 bottlenecks (the effect of a contraction or a bottleneck on nucleotide diversity is the same

153 provided that the bottleneck is not yet finished, or that it finished very recently so that the

154 effect of population recovery is negligible). In the present work, we investigate the effect of

155 short contractions on the genetic diversity and make the claim that this short contraction

156 regime is of particular interest as it can lead, such as in fig. 1c, to genomic signatures similar to 
157 those generated by positive selection acting on a few sites in an otherwise neutral genome.

158 More specifically, we want to quantitatively describe the reduction of diversity along the

159 genome that is observed around a locus with a small $T_{\text {MRCA }}$ (such as in fig. $1 \mathrm{c}$ in the regions

160 around $10-11$ and $19-20 \mathrm{Mb}$ ), where we observe a valley or trough of diversity. Akin to what is

161 done for selective sweeps, we consider the (neutral) fast fixation of an allele and analyze the

162 impact of hitchhiking on the genetic diversity of neighboring loci, and we refer to this process as

163 a neutral sweep.

164 To investigate neutral sweeps in our model, we consider the following scenario: $t_{\mathrm{m}}$ generations

165 ago a mutation occurred at a single site on the chromosome, which we call the focal site. We

166 further assume that this mutation has just fixed in the population, i.e., that it was segregating at

167 a frequency strictly lower than one in the last generation (at $t=1$ ), and has now (at $t=0$ ) a

168 frequency equal to one. We assume that the population contraction occurred $t_{c}$ generations

169 ago, with $t_{c} \geq t_{\mathrm{m}}$. As the mutant enters the population as a single allelic copy at the focal locus,

170 defined as a non-recombining region surrounding the focal site, this copy is a common ancestor

171 for all the copies $\left(2 N_{c}\right)$ present at fixation. However, it is not necessarily the most recent

172 common ancestor. Fig.2 shows a sketch of our model to help visualize how recombination can

173 maintain diversity at linked loci around a locus where a new mutation quickly fixed in the

174 population. 


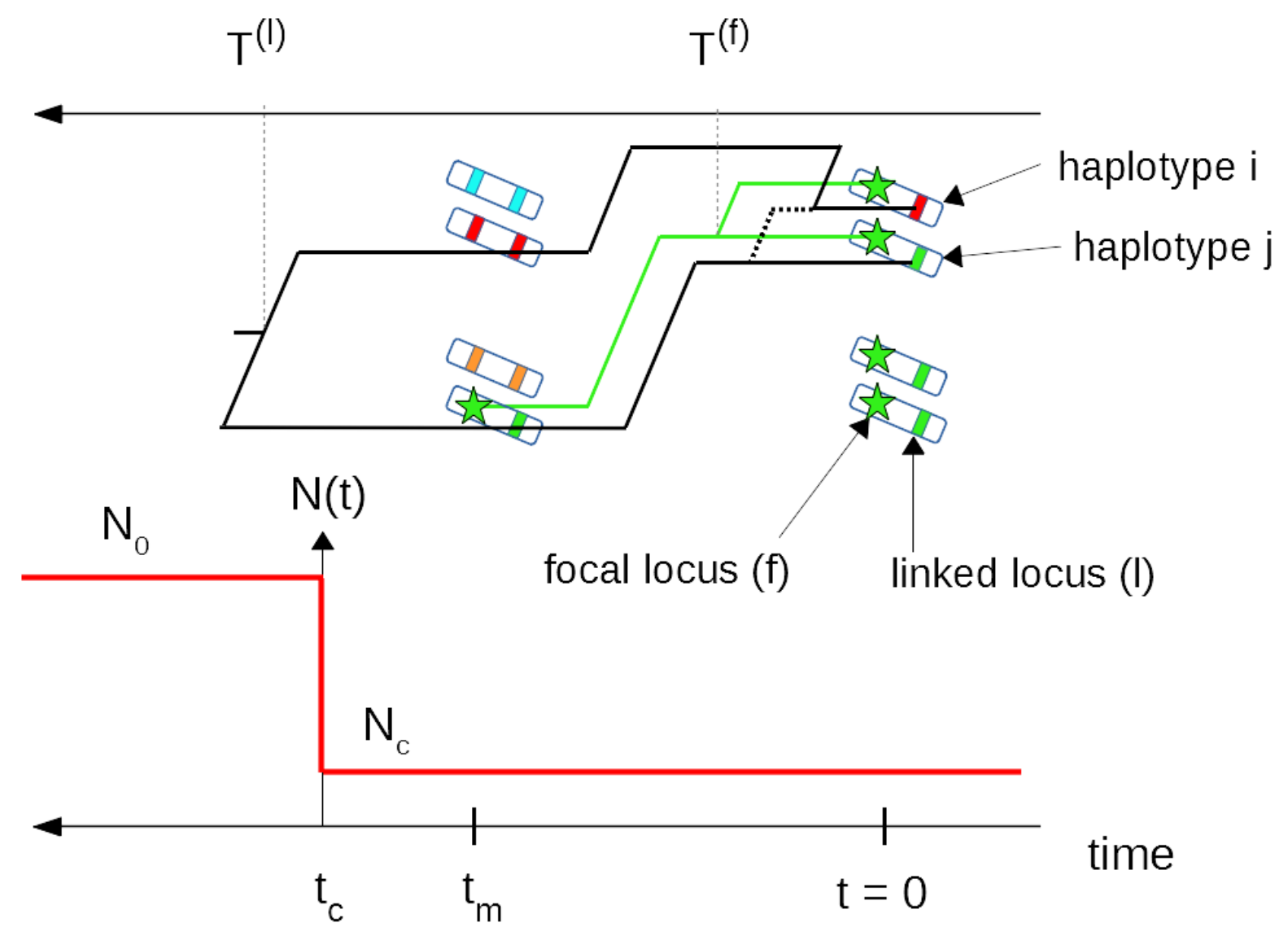

Figure 2. Instantaneous population contraction with a subsequent neutral fixation. A mutant (green star) appeared $t_{m}$ generations ago and has just fixed neutrally in a diploid population that experienced a contraction $t_{c}$ generations ago. We represent the population as a set of $2 N_{c}$ two-locus haplotypes that are painted so that the gene copies present at $t=0$ can be traced back to $t=t_{m}$. Due to recombination, haplotype i carries a red gene copy at the linked locus at $t=0$. Correspondingly, the coalescence time $T^{(I)}$ of the haplotypes $i$ and $j$ at the linked locus (black tree) is larger than $t_{m}$. On the other hand, the coalescence time $T^{(f)}$ at the focal locus 183 (green tree) is smaller than $t_{m}$ because at this locus all gene copies descend from the same 184 haplotype (due to the fixation of the focal mutation).

\section{Results}

187 We can calculate the expected coalescence time $T^{(1)}$ of two randomly sampled haplotypes at a

188 linked locus as a function of the recombination rate $r$ from the focal locus. The idea is to

189 consider two haplotypes with a given coalescence time $T^{(f)}$ at the focal locus, and then follow 
190 the genealogy of the gene copies carried by these two haplotypes at the linked locus backward

191 in time, while considering possible recombination events. The expected coalescent time at the

192 linked locus is then

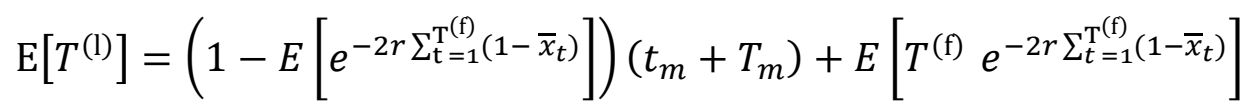

194 where $\bar{x}_{t}$ is the average frequency of the mutant (derived) allele at the focal locus at time $t$

195 counting backward from present. A detailed derivation of this equation is given in Appendix A4.

196 The first term of the right-hand side of eq. (2) corresponds to cases where lineages escape the

197 neutral sweep due to recombination, and still have not coalesced after $t_{\mathrm{m}}$ generations. In this

198 case we need to wait on average $T_{m}=2\left(N_{0}-N_{c}\right) e^{-\left(t_{c}-t_{m}\right) / 2 N_{c}}+2 N_{c}$ extra generations

199 before the lineages coalesce, due to the contraction that happened $t_{\mathrm{c}}-t_{\mathrm{m}}$ generations before

200 the focal mutation. The second term of the right-hand side of eq. (2) corresponds to cases

201 where the lineages cannot escape the sweep and are forced to coalesce at a time $T^{(l)} \leq t_{\mathrm{m}}$.

\section{Distribution of coalescence times at the focal locus}

203 To evaluate eq. (2), we need to determine the probability distribution of the pairwise

204 coalescence times $T^{(f)}$ at the focal locus, as well as the expected frequency trajectory of the

205 derived allele. Even though this allele fixes neutrally in a population of constant size (the

206 contraction occurs prior to the mutation), the distribution of coalescent times at the focal locus

$207 T^{(f)}$ departs from the usual exponential distribution for a neutral coalescent process because the

208 allele fixes in exactly $t_{\mathrm{m}}$ generations, and hence the coalescence time for a randomly chosen

209 pair of haplotypes is at most $t_{\mathrm{m}}$. Slatkin (1996) investigated the coalescent process within a

210 "mutant allelic class" that originated from a single mutation at a given time in the past. He

211 derived exact analytical results for the average pairwise coalescence time, but the coalescence

212 distribution itself can only be expressed with multidimensional integrals and obtaining a closed

213 form expression does not appear feasible. We therefore use a different approach: given a

214 particular fixation trajectory of the mutant allele, i.e. given the number of mutant copies $N_{\mu}$ at

215 each generation between $t=0$ and $t=t_{\mathrm{m}}$, we can express the coalescence time distribution

216 within the mutant allelic class, using the result of a coalescent in a population with a time- 
217 dependent (but deterministic) size $N_{\mu}(t)$ (Griffiths and Tavaré 1994). Averaging over all 218 possible trajectories of the mutation, we obtain:

$$
\mathrm{P}\left(\mathrm{T}^{(\mathrm{f})}\right)=\sum_{\left\{x_{t}\right\}}\left[\frac{1}{2 N_{c} x_{\mathrm{T}^{(\mathrm{f})}}} \prod_{t=1}^{\mathrm{T}^{(\mathrm{f})}-1}\left(1-\frac{1}{2 N_{c} x_{t}}\right)\right] P\left(\left\{x_{t}\right\}\right)
$$

220 where $x_{t}=N_{\mu}(t) /\left(2 N_{c}\right)$ is the frequency of the mutant $t$ generations from fixation, and

$221 P\left(\left\{x_{t}\right\}\right)$ is the probability of a given trajectory. $P\left(\left\{x_{t}\right\}\right)$ can be evaluated (see Appendix A2) and

222 the sum in eq. (3a) can in principle be computed numerically; however, the number of

223 trajectories to consider is prohibitive. As a first approximation, we can replace $x_{t}$ by its

224 expectation $\bar{x}_{t}$, i.e., we neglect the fluctuations of the trajectory around the mean to obtain

$$
\mathrm{P}\left(\mathrm{T}^{(\mathrm{f})}\right) \simeq \frac{1}{2 N_{c} \bar{x}_{\mathrm{T}^{(\mathrm{f})}}} \prod_{t=1}^{\mathrm{T}^{(\mathrm{f})}-1}\left(1-\frac{1}{2 N_{c} \bar{x}_{t}}\right) .
$$

226 The last step is to determine the average trajectory of an allele fixing in exactly $t_{\mathrm{m}}$ generations.

227 Zhao et al. (2013) as well as Maruyama and Kimura (Maruyama and Kimura 1975) have

228 investigated the characteristic trajectory of an allele fixing in a given time but they do not

229 provide a closed form solution. Here, we use a different approach (also based on diffusion

230 theory to obtain an approximation for the average trajectory of an allele fixing in exactly $t_{\mathrm{m}}$

231 generations, starting from a frequency $p_{0}$. As detailed in the Appendix $A 2$, we obtain

$$
\bar{x}_{t}=1 / 2\left(1-\left(1-2 p_{0}\right) e^{-\left(\mathrm{t}_{\mathrm{m}}-t\right) / N_{c}}+e^{-t / N_{c}}\right),
$$

233 which is valid for $t_{\mathrm{m}} \gg 2 N_{c}$. For very fast fixations, i.e., when $t_{\mathrm{m}} \ll 2 N_{c}$, the frequency of the

234 allele increases approximately linearly as

$$
\bar{x}_{t}=1-\left(1-p_{0}\right) \frac{t}{t_{m}}
$$

236 We remind the reader that $t$ is counted backwards from fixation. Fig. 3 compares equations (4a) 237 and $(4 b)$ to trajectories obtained from simulations of a Wright-Fisher diploid population. We

238 find good agreement between the simulations and the analytical results. Importantly, the 239 typical neutral trajectory for large values of the fixation time has an "inverse-sigmoid shape"

240 (fig. 3c), contrary to the typical sigmoid trajectory of a positively selected allele going to fixation 241 in a constant size population (see fig. 5a). This neutral trajectory occurs because, conditional on 
242 non-loss, neutral alleles need to quickly escape loss at the beginning and remain at

243 intermediate frequencies to stay away from both fixation and loss until they eventually fix in

244 the population at $t=0$ (i.e. in exactly $t_{\mathrm{m}}$ generations). Fig. $3 \mathrm{~d}-3 \mathrm{f}$ also shows the coalescence

245 time distribution for several values of the fixation time $t_{\mathrm{m}}$. The comparison of the distribution of

246 pairwise coalescence time with numerical simulations of a Wright-Fisher model shows that our

247 approximation eq. (3b) is quite accurate but overestimates the probability of coalescence for

248 large coalescence times when $t_{\mathrm{m}}$ is small (fig. $3 \mathrm{~d}$ ). Notably, coalescence (simulated or

249 theoretical) is more probable at large times (i.e. when the mutant appeared) for short fixation

250 times (fig. 3d), whereas it is more probable at small times (i.e. close to fixation) for large

251 fixation times (fig. 3e). The coalescence rate within the mutant allelic class is given by the

252 inverse of the number of mutant copies and is for all values of the fixation time slightly more

253 than $1 / 2 N_{c}$ at the first generation. However, when the fixation time is short (fig. $3 d$ ), there is a

254 fast increase of the coalescence rate backwards in time, and many lineages are forced to

255 coalesce at $t=t_{\mathrm{m}}$. When the fixation time is large (fig. 3f), the coalescence rate also increases

256 backwards in time, but the increase is much slower. In that case, most coalescence events

257 happen in much less than $t_{\mathrm{m}}$ generations, so that the early increase in frequency of the mutant

258 has almost no influence on the coalescence distribution.
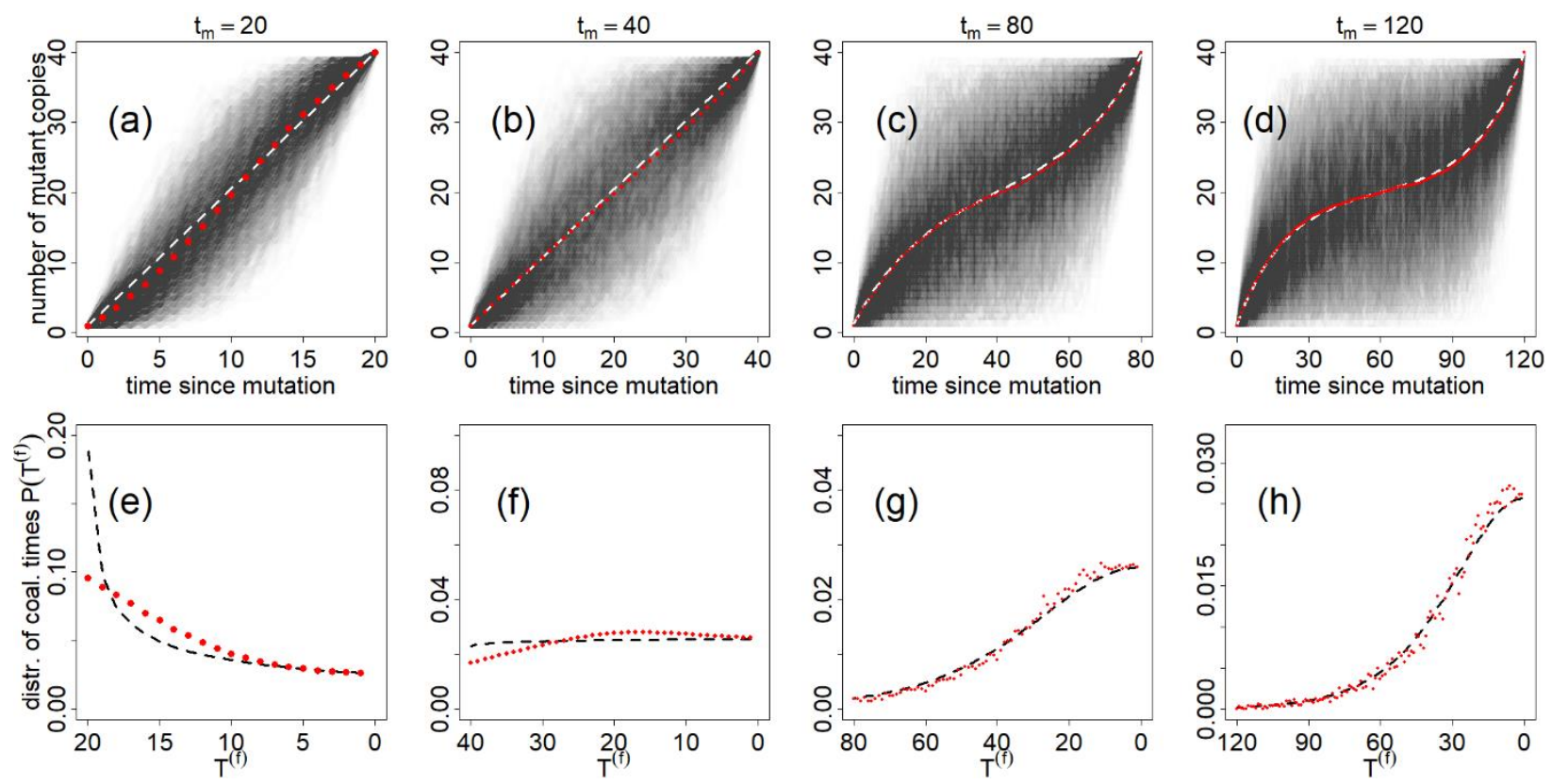

260 Figure 3. Average frequency $(a-d)$ and coalescence time distribution (e-h) of an allele fixing in a 261 diploid population of constant size $N_{c}=20$ in exactly $t_{m}$ generations, starting as a single copy 
(i.e. $\left.p_{0}=\left(2 N_{c}\right)^{-1}\right)$. The red dots are the results of Wright-Fisher simulations, and the black and white dashed lines are calculated with eqs. (4b) (first and second columns) (4a) (third and fourth columns) and (3b). In panes (a-d) we show the variability of the fixation process by overlapping 1780 fixing trajectories. The (numerically estimated) probability, for a mutant that appears at the onset of the contraction, to fix in less than $t_{m}$ generations is $0.006,0.16,0.64$ and 0.86 for $t_{m}=20,40,80$ and 120 respectively (for this particular value of $N_{c}$ ).

\section{Effect of a neutral sweep on linked diversity}

269 Combining equations (3b), (4a) with eq. (2) allows us to get an approximation for the average coalescence time at linked loci. Since the derivation of eq. (2) assumes that there is at most one recombination event in the genealogy of a randomly chosen pair of gene copies, we expect it to

272 be only accurate for small values of the recombination rate $r$. For large values of $r$ we use a 273 heuristic approach combining the result of eq. (2), which is accurate for small $r$, and the 274 expected diversity at unlinked loci, which is equal to $T_{0}=2\left(N_{0}-N_{c}\right) e^{-t_{c} / 2 N_{c}}+2 N_{c}$ as stated 275 in eq. (1). We fit the trough of diversity with an exponential function of the form:

$$
\mathrm{E}\left[\mathrm{T}^{(1)}\right](r)=T_{0}\left(1-c e^{-a r}\right),
$$

277 where the coefficients $c=1-E\left[\mathrm{~T}^{(\mathrm{f})}\right] / T_{0}$ and $a=2 E\left[\left(t_{m}+T_{m}-\mathrm{T}^{(\mathrm{f})}\right) \sum_{t=1}^{\mathrm{T}^{(\mathrm{f})}}\left(1-\bar{x}_{t}\right)\right] /\left(T_{0}-\right.$ $278 E\left[\mathrm{~T}^{(\mathrm{f})}\right]$ ) are obtained by imposing that eqs. (2) and (5) coincide for small values of $r$ (using a

279 linear expansion in $r$ ). On fig. 4 we compare the result of eq. (5) to Wright-Fisher simulations

280 with two recombining loci. We see in fig. 4a that the exponential function fits the data accurately 281 at large values of the recombination distance, but that the fit is biased for intermediate values of $282 r$. In fig. 4b we see that the approximation is very good for low values of the recombination 283 distance, although there still is a slight bias. This discrepancy at small $r$ can be corrected (solid 284 lines in fig. 4) if we use numerical estimations of $\bar{x}_{t}$ and $\mathrm{P}\left(\mathrm{T}^{(\mathrm{f})}\right)$, instead of eqs. (4) and (3b), to 

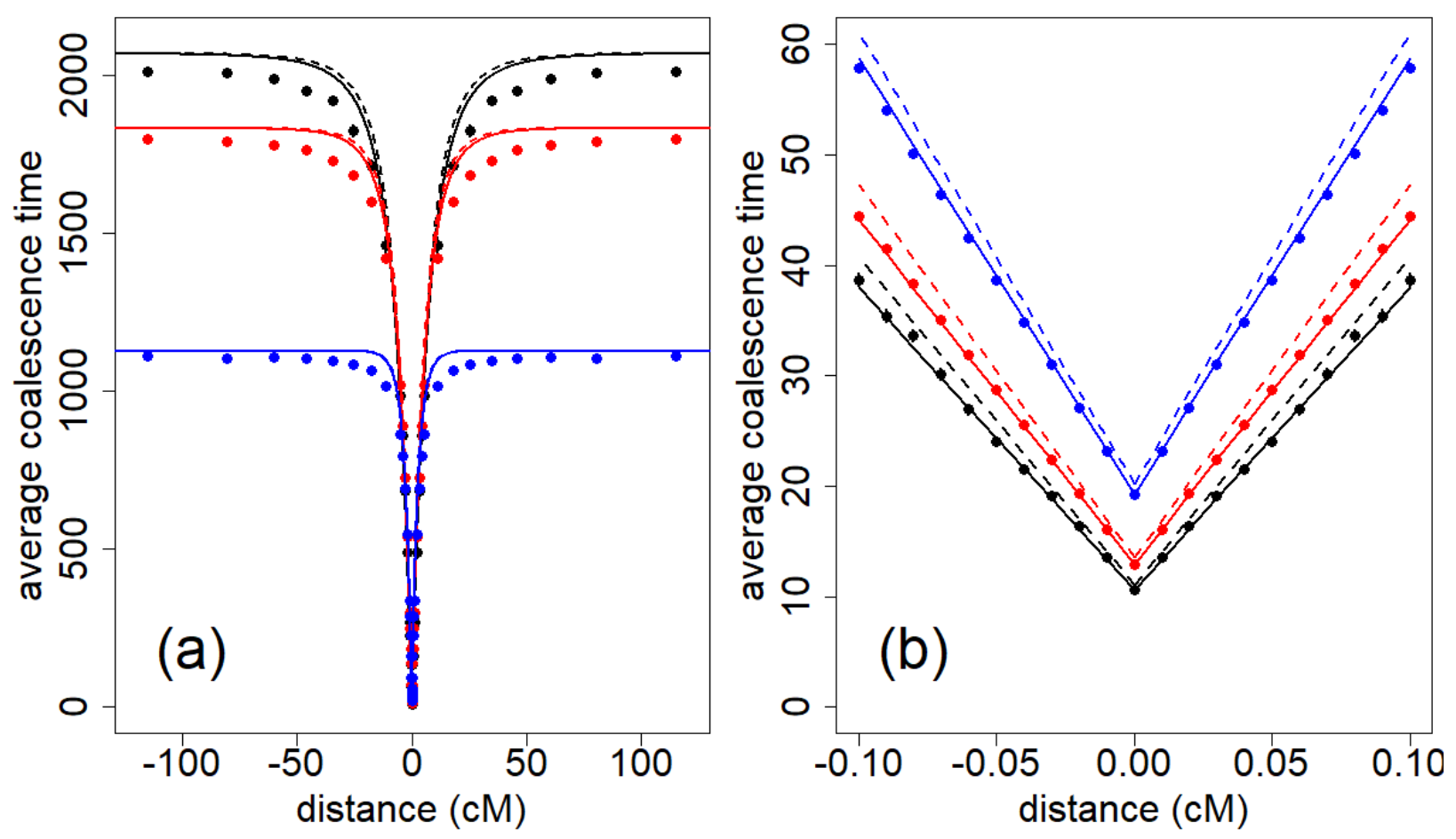

Figure 4. Average coalescence time at a linked locus, as a function of the recombination distance from the focal locus where a mutant fixed in exactly $t_{m}$ generations, starting from a single copy $t_{m}$ generations ago. $t_{m}=15$ in black, $t_{m}=20$ in red and $t_{m}=40$ in blue. The dots are calculated with two-locus WF simulations, and compared to eq. (5) with either a numerical estimation (solid lines) or a theoretical estimation (dashed lines) of $\bar{x}_{t}$ and $P\left(T^{(\mathrm{f})}\right) . N_{c}=20 . N_{0}=$ 1500. The population experienced a contraction $t_{c}=t_{m}$ generations ago.

We observe, as expected, on fig. 4 that the troughs of diversity induced by neutral sweeps are wider and deeper for short fixation times. Similarly to what happens after a selective sweep, there is less opportunity for linked loci to escape the sweep by recombination and maintain

297 diversity when the fixation is fast. In addition, the diversity level at the center of the valley is 298 given by the average coalescence time at the focal locus, which quickly decreases for small 299 fixation times $t_{\mathrm{m}}$.

Comparison of neutral sweeps and selective sweeps

301 Since we did not make any assumption regarding the process driving the mutant allele to

302 fixation when deriving the average coalescence time at linked loci (eq. (2)) and the coalescence 
303 time distribution at the focal locus (eq. (3b)), our framework allows us to directly compare the

304 signatures of different processes that can drive mutations to fixation in a given number of

305 generations. We illustrate this by comparing the effect of neutral and hard selective sweeps on

306 linked diversity. Later we will discuss how neutral sweeps compare to a larger variety of

307 scenarios (e.g. background selection, small selection coefficients, or dominant alleles). Here we

308 assume that the neutral and selected fixations occurred over the same time interval, that is in

309 both cases in exactly $t_{\mathrm{m}}$ generations. The selected fixation is assumed to be codominant $(h=0.5)$

310 and occurs on an autosomal locus in a randomly mating diploid population of constant size $N_{1}$,

311 and we consider a strong selection strength $\left(2 N_{1} S>>1\right)$ so that the allele frequency follows the

312 deterministic trajectory

$$
\bar{x}_{\mathrm{t}}=\frac{1}{1+\left(2 N_{1}-1\right) e^{-2\left(1-t / t_{\mathrm{m}}\right) \log \left(2 \mathrm{~N}_{1}\right)}},
$$

315 where the fixation time is given by $t_{\mathrm{m}}(s)=2 \log \left(4 N_{1} s\right) / s$ (Barton 1995). Then combining eqs. (5),

316 (3b) and (6), we can compute the average coalescence time at linked loci as a function of the

317 recombination distance $r$ to the focal locus, after replacing $T_{\mathrm{m}}$, the average coalescence time at $t$

$318=t_{\mathrm{m}}$, by $2 N_{1}$ in eq. (5) and $N_{\mathrm{c}}$ by $N_{1}$ in eq. (3b). This approach yields results similar to

319 Charlesworth (2020), where the author investigated signals of selective sweeps correcting for

320 coalescent events that happen during the sweep, thus going beyond the common assumption of a

321 star tree structure at the focal locus. For sake of simplicity in the neutral case, we consider that

322 the mutant appeared at the time of the contraction, i.e. $t_{\mathrm{m}}=t_{\mathrm{c}}$. Furthermore, we will assume that

323 the average coalescence times (and consequently the genetic diversity) are equal in both

324 scenarios, i.e. that $T_{0}=2 N_{1}$ which implies that 


$$
N_{0}\left(\mathrm{t}_{\mathrm{m}}\right)=\left(N_{1}-N_{c}\right) e^{t_{m} / 2 N_{c}}+N_{c} .
$$

326 In the neutral case we want the diversity to remain as high as $4 N_{1} \mu$ after the contraction, which

327 is possible only if the ancestral diversity was even higher, i.e. we have in general $N_{0}>N_{1}>N_{c}$.

328

329
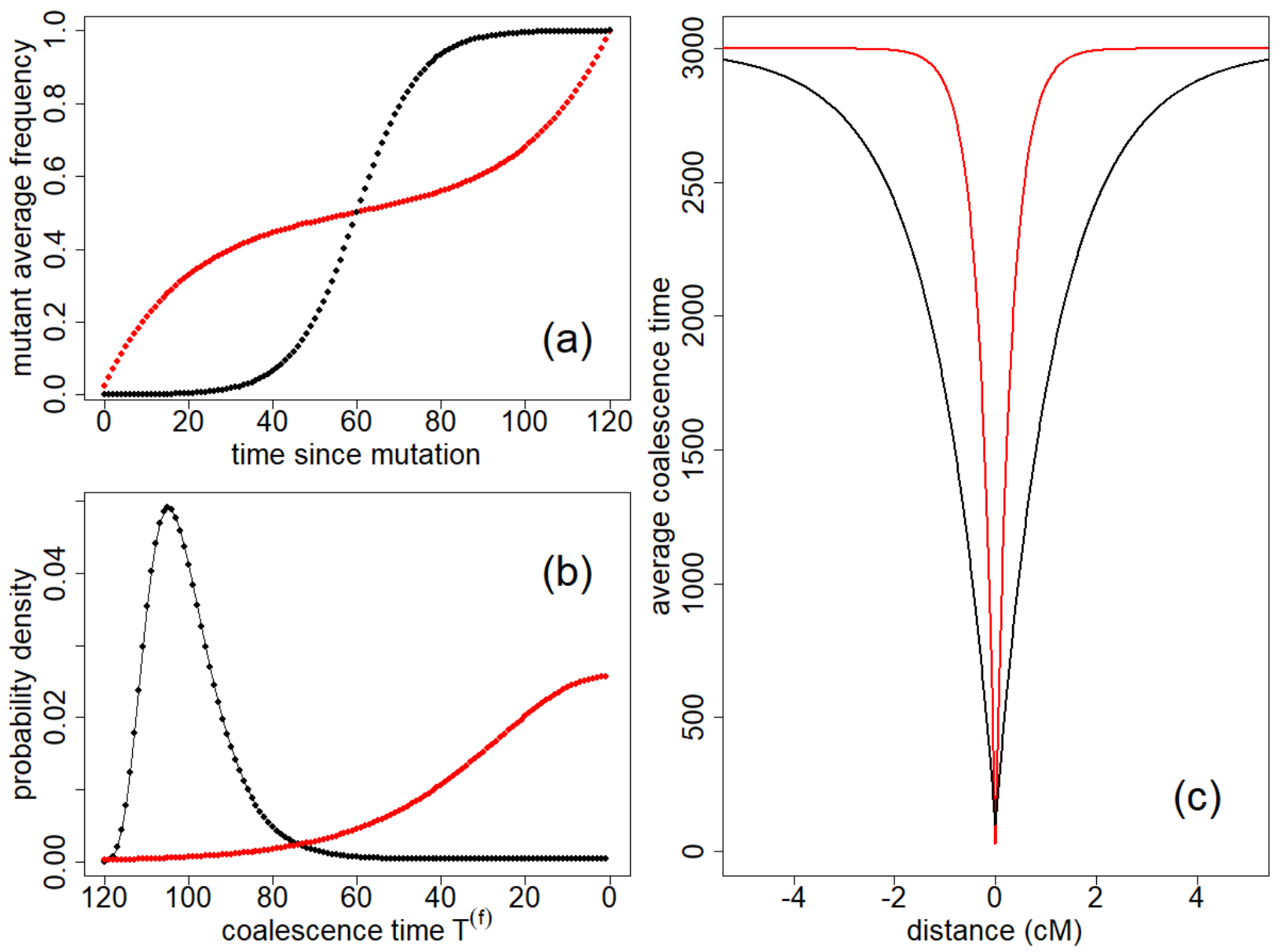

Figure 5. Comparison between troughs of diversity resulting from a selective sweep (black) and a neutral sweep (red), for the same fixation time $t_{m}=120$ (corresponding to $s \approx 0.1$ in the selective case). Frequency of the fixing allele as a function of time (a), coalescence time distribution (b) and diversity around the fixing site along the genome using eq. (5) (c). $N_{1}=1500$, $N_{c}=20$ and $N_{0}=2.97 \times 10^{4}$.

In fig. 5a, we compare the mutant average frequency as a function of time for a selected and a neutral fixation. The dynamics of the neutral fixation is the opposite of that of the selected allele in the sense that when one is increasing, the other is "resting" and vice versa. These different trajectories translate into different coalescence distributions at the focal locus (fig. 
$3405 b)$. If selection drives the fixation of the mutation, the distribution of coalescence time is

341 peaked at large coalescence times. In contrast, in the neutral case the distribution is skewed

342 towards small coalescence times. Correspondingly, the coalescence tree for the selected case

343 has a star-like structure (not shown), whereas the tree for the neutral case has shorter outer

344 branches. Therefore, for a given recombination distance, there will be fewer recombinations on

345 the neutral tree because it has a much smaller total length. As recombination helps maintain

346 diversity at linked loci, we would expect neutral troughs of diversity to be wider than in the

347 selected case. However, this is at odds with the valleys of diversity observed in fig. $5 c$, where

348 the selective trough is wider than the neutral trough. In fact, even though recombination is less

349 abundant on the neutral tree, it is more efficient at recovering diversity. Indeed, if at a linked

350 locus a pair of lineages escapes the sweep due to recombination, it takes on average an extra

$3512 N_{1}$ generations, counted backwards from generation $t=t_{\mathrm{m}}$ when the mutant appeared, for

352 them to coalesce in the selective case, and an extra $2 N_{0}$ generations in the neutral case. As $N_{0}>$

$353 \quad N_{1}$ two lineages escaping the sweep due to recombination have a larger coalescence time in the

354 neutral case, and correspondingly a larger diversity, which explains why the neutral valley of

355 diversity is narrower. Furthermore, we see that the trough is deeper in the neutral case (fig. $5 c)$,

356 since the average coalescence time is smaller at the focal site due to the smaller total length of

357 the coalescence tree.

359 To determine if these differences between selective and neutral troughs hold for other fixation

360 times and population sizes, we define two quantities that characterize the shape of a trough, as

361 well as its propensity to be detected in real data: i) the trough relative depth and ii) the width of

362 the trough. The relative depth is defined as the difference between the background level of

363 diversity and the diversity at the focal locus, divided by the background diversity, and the width

364 is measured at half depth, i.e. halfway between the background diversity and the diversity at

365 the focal locus. On fig. 6 we plot the relative depth of neutral and selective troughs as a

366 function of their width for different fixation times $t_{\mathrm{m}}$, calculated with our analytical expressions.

367 We see that the neutral troughs are not only always narrower than the selective troughs for the

368 same value of $t_{\mathrm{m}}$, but also deeper. This is due to differences in the focal tree structure between 
the selective case and the neutral case as well as difference in the ancestral background level in both cases, as explained above. For very short fixation times (corresponding to selection coefficients larger than 0.1 ), there is almost no difference between troughs generated by

372 selective and neutral sweeps. Indeed, for such values of $t_{\mathrm{m}}$, in both cases the focal coalescence

373 tree is essentially a star tree because the increase in frequency is very fast, and the ancestral

374 backgrounds of diversity, $2 N_{0}$ and $2 N_{1}$, are also practically equal. Note however that at small $t_{\mathrm{m}}$

375 the corresponding value of the selection coefficient $s$ (see legend of fig. 6) may be

376 unrealistically high. For realistic values of the selection coefficient/fixation time, the neutral

377 troughs tend to be quite deep but narrow, whereas selective troughs are wider and their depth

378 decreases quickly for low selection coefficients. From fig. 6, we see that the shape of a neutral

379 trough is generally different from a selective sweep signal, but in practice those differences

380 might be hidden due to the noise inherent present in real genomic data, and it might be

381 difficult to decide whether a genomic signal is a due to a neutral sweep or a selective sweep.

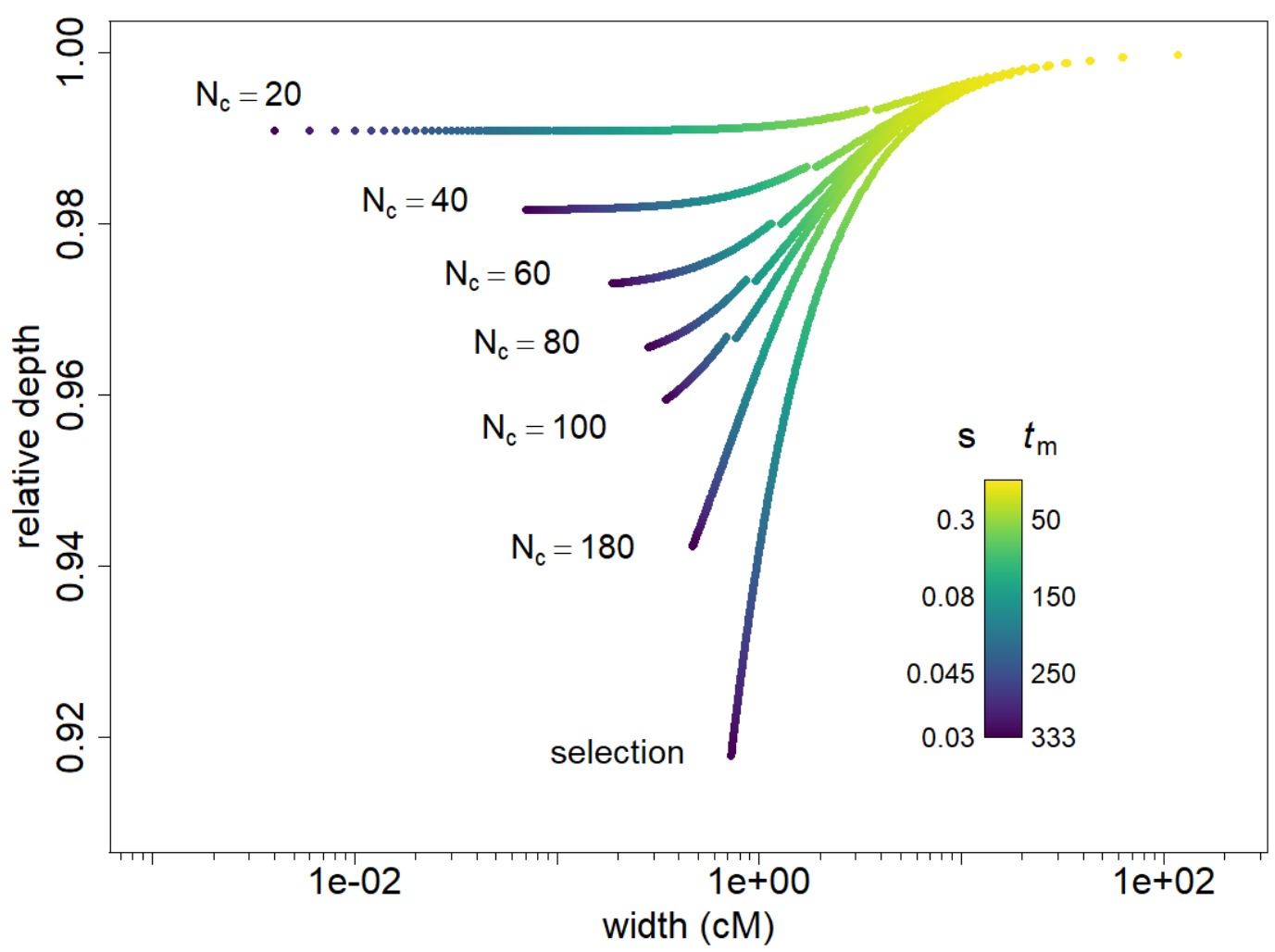

383 Figure 6. Relative depth as a function of the width of the diversity troughs, for different values

384 of $t_{m}$ and $N_{c}$ in the neutral case and for selective scenarios with identical fixation times. $t_{m}$ goes 385 from 1 to 333 by increments of 1, the corresponding values of the selection coefficient $s$ are 
indicated on the left of the legend bar (for all of them we have $\left.N_{1} s>1\right) . N_{1}=1500 . N_{0}$ is given by eq. (7) and depends on $N_{c}$ and $t_{m}$. The jumps in the neutral curves for $N_{c}=20,40,60,80$ and

388100 are due to the use of two different approximations for the frequency of the mutant, eqs.

$389(4 a)$ and $(4 b)$ and are located at $t_{m}=2 N_{c}$.

Is the Qtzl trough in D. melanogaster a neutral trough?

391 A region with reduced nucleotide diversity around the Quetzalcoat/ gene identified in

392 Drosophila melanogaster was judged compatible with a selective sweep (Rogers et al. 2010). A

393 hard sweep model (Kaplan et al. 1989) was fitted assuming a constant population size of $N_{1}=$

$3941.85 \times 10^{6}$ diploid individuals and it was inferred that a positively selected allele fixed in the

395 population $1.5 \times 10^{5}$ generations ago $\left(1.5 \times 10^{4}\right.$ years $)$ due to a selective advantage of $s=0.0098$

396 (corresponding to a fixation time of more than 300 years). Using our theory, we fitted the data

397 under a neutral demographic scenario of recent population size change that can generate

398 neutral troughs with the same width and almost the same relative depth (less than $0.1 \%$

399 difference) as the Quetzalcoatl trough. To infer the demographic parameters, we measure the

400 width of the selective sweep curve used to fit the data in (Rogers et al. 2010) and find a set of

401 values of $\left(N_{\mathrm{c}}, t_{\mathrm{m}}\right)$ that define a neutral trough with the same width. We then impose that $t_{\mathrm{m}} / 2 N_{\mathrm{c}}$

$402=0.25$ so that the troughs are rare yet observable along the chromosome as explained on fig. 1 ,

403 and we obtain $t_{\mathrm{m}}=2200$ and $N_{\mathrm{c}}=4400$. In fig. 7 we show a trough generated during a

404 population contraction corresponding to these inferred values, using the software fastsimcoal2

405 (Excofffier et al. 2021). We see that the neutral sweep fit is almost indistinguishable from the

406 selective sweep fit because they not only have the same width, but also practically the same

407 depth. Note that this simulated trough can be also seen in fig. $1 \mathrm{c}$ in the region 19-20 Mb. The

408 same approach can be used to generate neutral troughs with a broad range of width and depth,

409 which implies that in most cases, an alternative demographic neutral scenario can be

410 compatible with a trough that is putatively due to selection. In practice, model inference does

411 not rely solely on the fitting of a single trough, and genome wide information must be used.

412 Therefore, we do not exclude here the possibility of the presence of adaptation in the

413 Quetzalcoatl gene, but rather make the general warning that valleys of diversity do not

414 necessarily indicate the presence of positive selection. 
415 The authors affirm that all data necessary for confirming the conclusions of the article are present within the article, figures, and tables.

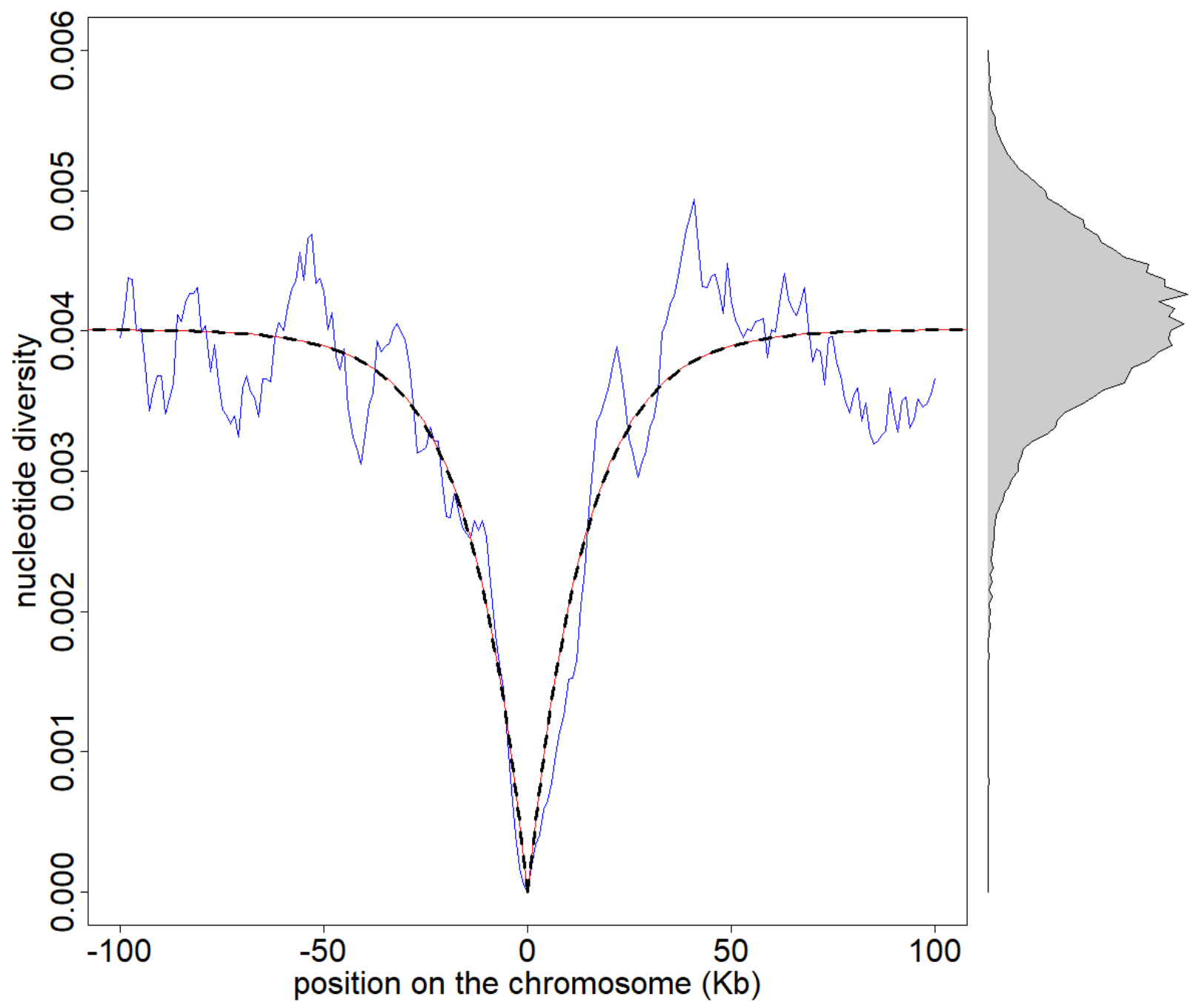

418 Figure 7. Trough of nucleotide diversity observed on a $20 \mathrm{Mb}$ chromosome simulated with 419 fastsimcoal2. The population experienced a contraction 2200 generations ago and the (diploid) 420 population size was reduced from $N_{0}=2.37 \times 10^{6}$ to $N_{c}=4400$. The nucleotide diversity (blue line) 421 is calculated on a sample of 30 haplotypes from our simulation. The black dashed line is the 422 expected diversity (eq. (5)) for an allele that just fixed neutrally in the population, starting as a 423 single copy 2200 generations ago. The red line is the expectation of a hard selective sweep with 424 selection coefficient $s=0.0098$. On the right we plot the distribution of nucleotide diversity for 425 the whole chromosome. The mutation rate $\mu=5.42 \times 10^{-10}$ per site per generation, and 426 recombination rate $r=3.5 \times 10^{-8}$ per site per generation were taken from (Rogers et al. 2010). 427 The nucleotide diversity (in blue) is calculated for sliding windows of $10 \mathrm{~Kb}$ at $1 \mathrm{~Kb}$ intervals. 


\section{Discussion}

429 It has repeatedly been suggested that strong depletions of diversity in the genome are not

430 necessarily due to the presence of positive selection (Johri et al. 2020), and can also be the

431 result of demographic effects only, such as the allele surfing phenomenon occurring at the front

432 of a range expansion (Klopfstein et al. 2006). In this work, we considered a model of population

433 contraction to analyze quantitatively the genomic signature of the rapid fixation of a mutation

434 during a population contraction. Taking a step further from previous work that focused on the

435 impact of range expansion on mere allele frequencies, we have studied here the impact of a

436 neutral allele fixation on neighboring genomic diversity. We show that the diversity profile

437 around a recently fixed locus crucially depends on the frequency trajectories of the allele going

438 to fixation, and we outline the fact that neutrally fixing alleles have an inverse-sigmoid

439 trajectory (fig. 3c), as compared to the standard sigmoid frequencies observed for positively

440 selected alleles. For the same fixation time, this difference translates into different genomic

441 signatures (see figs. $5 \mathrm{c}$ and 6). Our results demonstrate that there is a short period after a

442 demographic contraction (or during a range expansion) where observed profiles of genomic

443 diversity would look like those usually attributed to selection (fig. 1c), and that selective sweep

444 signals can be mimicked by neutrally fixing mutations without the need to invoke complex

445 histories of population size changes.

446 Our results allow for a systematic comparison of selective and neutral troughs of diversity, and

447 we used our results to investigate trough shapes for range of neutral and selected scenarios

448 (see fig. 6), which in principle can be used to decide whether a given empirical trough is due to

449 selection or demography, and to infer the corresponding parameters. However, we did not

450 consider the whole spectrum of possible selection scenarios. It would be indeed interesting to

451 use our results to study cases of background selection, small selection coefficients, and a

452 variety of dominance coefficients. All these cases should have their own characteristic

453 trajectories of fixation, and hence potentially different genomic signatures. In addition, in our

454 model we do not consider mutations that fixed in the past (we always assume that the allele

455 has just reached fixation), nor do we consider mutations appearing before the population

456 contraction, i.e., with $t_{\mathrm{m}}>t_{\mathrm{c}}$. The average coalescence time in the former case can be expressed 
457 as a function of the coalescence time at fixation using conditional probabilities, and we can

458 show that a sweep signal vanishes exponentially with the time elapsed since fixation (see

459 Appendix A4). In the latter case, we can solve the problem by considering the number of gene

460 copies at $t_{\mathrm{c}}$ that descend from the original copy that appeared at $t_{\mathrm{m}}$. One could extend our

461 results by considering an allele starting from an arbitrary number of copies at $t_{c}$, akin to soft

462 selective sweeps; however, the analytic calculations are complex, and we leave this study for

463 future research. In any case, those additional scenarios must be considered when trying to infer

464 models from the study of troughs found in empirical data. Another phenomenon that renders

465 the inference of parameters cumbersome is a possible interference between troughs. Indeed,

466 when two loci fix neutrally in the population, the genetic diversity in the region between those

467 loci will be influenced by both fixations and will differ from the diversity expected in the vicinity

468 of a single fixing locus. As in the case of interference between the fixation of selected alleles

469 (Weissman and Barton 2012), this should limit the number of independent neutral fixations.

470 The effect of trough interference is stronger for neighboring troughs, and the probability to

471 observe close troughs depends on the relative frequency of troughs along the genome, which

472 itself depends on the distribution of the $T_{M R C A}$. In fig. $1 \mathrm{~d}$ for example, the distribution of $T_{M R C A}$

473 has a mode centered around $4 N_{c}$ (not shown) and correspondingly the nucleotide diversity is

474 peaked around $4 N_{c} \mu$. As a result, we see many regions of the chromosome with a low diversity.

475 It is likely that those troughs interfere with each other and that they do not correspond to the

476 profile of an isolated trough. On the other hand, in fig. 1c, the first mode of the $T_{M R C A}$

477 distribution is truncated because $t_{\mathrm{c}}$ is much smaller than $4 N_{c}$, and only $T_{M R C A} \mathrm{~s}$ equal or close to

$478 t_{\mathrm{c}}$ are observed (plus all the $T_{M R C A} \mathrm{~s}$ corresponding to the second mode centered at $4 N_{0}$ ). In this

479 case there is no interference and the (rare) troughs, such as the one in fig. 7, are correctly fitted

480 by their theoretical expectation. Those considerations imply that, even though we know the

481 forward in time probability that an allele will fix in $t_{m}$ generations, it is difficult to infer the

482 parameters of a fixation scenario from a single observed neutral valley of diversity. It appears

483 therefore difficult to perform model selection from a single trough signal, i.e., to decide

484 whether a particular trough is due to selection or demographic effects, because alternative

485 demographic scenarios that we did not consider here could also lead to similar signals. In 
principle, if several troughs of diversity were observed in a genome, one could use the distribution of trough shapes expected under a given simple demographic model and a distribution of fitness effect to compare neutral and selection models under a likelihood framework.

In conclusion, our results suggest that any empirical valley of diversity found in empirical data can be reproduced neutrally with a population contraction using appropriate parameters. One could argue that this identifiability problem disappears once the true evolutionary history is correctly inferred. However, inferring the true demographic history requires precise knowledge 494 about how selection has shaped genomic diversity (Johri et al. 2020). In humans, for instance, it 495 has been estimated that roughly $95 \%$ of genomic diversity is affected by some form of nonneutral forces such as background selection or biased gene conversion (Pouyet et al. 2018)

497 potentially biasing demographic inference (Ewing and Jensen 2016). These considerations 498 indicate than genome scans in search for signals of adaptation might be subject to stronger 499 false positive rates than previously thought. We thus believe that despite current advances 500 using supervised machine learning or similar approaches (Schrider and Kern 2018), it remains

501 important to further study the effect of neutral fixations in various demographic scenarios using 502 localized genomic approaches such as the present analytical work (Johri et al. 2021b); as well as 503 with controlled experiments on real living organisms where both the selected locus and the 504 population history are known (Orozco-terWengel et al. 2012). Such work will be critical in order 505 to develop more appropriate evolutionary null models for statistical inference (Hahn 2008;

506 Johri et al. 2020).

\section{Appendix}

\section{A1. Coalescence distribution after a contraction}

509 We want to determine the coalescence time of two lineages in a population that experienced a 510 contraction $t_{m}$ generations ago, from a diploid size $N_{0}$ to $N_{c}$. As we go backward in time, the 511 coalescence rate switches from $\left(2 N_{c}\right)^{-1}$ to $\left(2 N_{0}\right)^{-1}$ at $T=t_{\mathrm{c}}$. The probability distribution might

512 still be approximated by a piecewise exponential density: 


$$
\begin{aligned}
f_{0}(T) & =\frac{1}{2 N_{c}} \exp \left(-\frac{T}{2 N_{c}}\right) \text { for } 0<T<t_{\mathrm{c}} \\
& =\frac{1}{2 N_{0}} \exp \left(-\frac{t_{\mathrm{c}}}{2 N_{c}}\right) \exp \left(-\frac{T-t_{\mathrm{c}}}{2 N_{0}}\right) \text { for } T \geq t_{\mathrm{c}}
\end{aligned}
$$

514 The corresponding expectation for this distribution is

$$
\begin{aligned}
E[T]=T_{0} & =\int_{0}^{\infty} T f_{0}(T) \mathrm{d} T \\
& =2 N_{0} e^{-t_{c} / 2 N_{c}}+2 N_{c}\left(1-e^{-t_{c} / 2 N_{c}}\right)
\end{aligned}
$$

\section{A2. Average frequency of an allele fixing in exactly $t_{\mathrm{m}}$ generations}

517 In this section time is counted forward from the mutation, which appears after the contraction,

518 so that during the fixation the diploid population size is constant and equal to $N_{c}$. We condition

519 on the fixation time $t_{\mathrm{m}}$ of the mutant. We define the trajectory of a mutant as the list of

520 frequencies at all generations: $\left\{x_{t}\right\}=\left(x_{0}, x_{1}, \ldots, x_{t_{m}-1}, x_{t_{m}}\right)$. We assume that the mutant fixes

521 in exactly $t_{\mathrm{m}}$ generations, starting from a frequency $p_{0}$, i.e. $x_{0}=p_{0}, 0<x_{t_{m}-1}<1$ and $x_{t_{m}}=$

522 1. The probability that the mutant follows a given trajectory might be expressed as the product

523 of the transition probabilities

$$
\mathrm{P}\left(\left\{x_{t}\right\}\right)=\prod_{t=0}^{t_{\mathrm{m}}-1} P\left(i, t \rightarrow j, t+1 \mid \text { fix in } t_{m}, p_{0}\right)
$$

525 For an unconditional Wright Fisher model, $\mathrm{P}(\mathrm{i}, \mathrm{t} \rightarrow \mathrm{j}, \mathrm{t}+1)$ is the probability to have $\mathrm{j}$ copies of 526 the new allele at $t+1$ given that there were i copies at $t$. We note $P_{t}(i \rightarrow j)$ for brevity. If we 527 only consider trajectories fixing in exactly $t_{m}$ generations and starting from a number $2 N_{c} p_{0}$ of 528 copies at $\mathrm{t}=0$, then the transition probabilities are not equal to the transitions of the 529 unconditional Wright-Fisher model. However, thanks to Bayes theorem, we can write

$$
\begin{aligned}
P_{t}\left(i \rightarrow j \mid \text { fix in } t_{m}, p_{0}\right) & =\frac{P_{t}\left(\text { fix in } t_{m} \mid i \rightarrow j, p_{0}\right) P_{t}\left(i \rightarrow j \mid p_{0}\right)}{P\left(\text { fix in } t_{m} \mid p_{0}\right)} \\
& =\frac{P\left(\text { fix in } t_{m} \mid j_{t+1}\right) P_{t}(i \rightarrow j)}{P\left(\text { fix in } t_{m} \mid p_{0}\right)}
\end{aligned}
$$


531 From the first to the second line, we use the Markov property. The three terms involved in the

532 right-hand side of this equation can be approximated thanks to diffusion theory. In this

533 framework, the probability for an allele to fix in $t_{m}$ generations, given that there were i copies

534 at time $t$ is approximately (Ewens 2004, taking the time derivative of eq. 5.39)

$$
\mathrm{P}\left(\text { fix in } t_{m} \mid i_{\mathrm{t}}\right)=\frac{3}{2 N_{c}}\left(1-\frac{i}{2 N_{c}}\right) \frac{i}{2 N_{c}} e^{-\left(t_{m}-t\right) / 2 N_{c}}
$$

The term $P_{t}(i \rightarrow j)$ is the unconditional binomial transition probability of the Wright Fisher model (which does not depend on t). In principle, eq. (S1) can be used to compute the exact distribution of coalescence times at the focal locus, using eq. (3a). However, the huge number of possible trajectories fixing in $t_{m}$ generations $\left(\left(2 N_{c}-1\right)^{t_{m}-1}\right)$ makes the average over trajectories impossible to evaluate numerically. For this reason, we use the approximation in eq. (3b).

542 We consider here the probability that the allele has frequency $\mathrm{x}$ at time $\mathrm{t}$, given that it started 543 at frequency $p_{0}$ at $\mathrm{t}=0$. Again if we only consider trajectories that fix in exactly $t_{m}$ 544 generations, this probability is not equal to the neutral diffusive result. However, similarly to 545 the previous section, we can use Bayes theorem:

$$
\mathrm{P}\left(x_{t} \mid \text { fix in } t_{m}, p_{0}\right)=\frac{P\left(\text { fix in } t_{m} \mid x_{t}\right) P\left(x_{t} \mid p_{0}\right)}{P\left(\text { fix in } t_{m} \mid p_{0}\right)}
$$

$$
\mathrm{P}\left(x_{t} \mid p_{0}\right)=6 p_{0}\left(1-p_{0}\right) e^{-t / 2 N_{c}}\left(1+5\left(1-2 p_{0}\right)(1-2 x) e^{-t / N_{c}}\right)
$$

549 which is a second order expansion of an infinite series involving vanishing exponential terms $550\left(e^{-k(k+1) t / 4 N_{c}}\right.$ for all $\left.\mathrm{k} \geq 1\right)$. This expansion is thus valid in the limit of large times $\mathrm{t} \gg 2 N_{c}$. We 551 deduce that the probability that an allele fixing in $t_{m}$ generations has frequency $\mathrm{x}$ at time $\mathrm{t}$ is

$$
\mathrm{P}\left(x_{t} \mid \text { fix in } t_{m}, p_{0}\right)=6 \mathrm{x}(1-x)\left(1+5\left(1-2 p_{0}\right)(1-2 x) e^{-t / N_{c}}\right)
$$

$$
\text { which yields } \mathrm{E}\left[x_{t} \mid \text { fix in } t_{m}, p_{0}\right]=1 / 2\left(1-\left(1-2 p_{0}\right) e^{-t / N_{e}}\right)
$$


554 This expression is valid for $t_{m} \gg t \gg 2 N_{c}$, and does not allow to estimate the frequency close

555 to fixation (we see that $\mathrm{E}\left[x_{t}\right]$ tends to $1 / 2$ as time grows). However, invoking a symmetry

556 argument we may write

$$
\mathrm{E}\left[x_{t} \mid \text { fix in } t_{m}, p_{0}\right]=1 / 2\left(1-\left(1-2 p_{0}\right) e^{-t / N_{c}}+e^{-\left(\mathrm{t}_{\mathrm{m}}-t\right) / N_{c}}\right)
$$

558 When $\mathrm{t}_{\mathrm{m}} \ll 2 N_{c}$, we can use a linear approximation for the trajectory (based on the numerical 559 observations)

$$
\mathrm{E}\left[x_{t} \mid \text { fix in } t_{m}, p_{0}\right]=p_{0}+\left(1-p_{0}\right) \frac{t}{t_{m}}
$$

\section{A3. Coalescence distribution at linked loci around a neutral fixation}

562

563

564

565

We now return to the scenario of fig. 2, with a backward in time approach. Using Bayes theorem, we express the coalescence time of two haplotypes at the linked locus $T^{(l)}$, conditioning on the coalescence time at the focal locus $T^{(f)}$

$$
\mathrm{P}\left(T^{(l)}\right)=\int_{0}^{\mathrm{t}_{\mathrm{m}}} \mathrm{P}\left(T^{(l)} \mid T^{(f)}\right) P\left(T^{(f)}\right) \mathrm{d} T^{(f)}=\mathrm{E}\left[\mathrm{P}\left(T^{(l)} \mid T^{(f)}\right)\right]
$$

We assume that the linked locus is close to the focal locus on the chromosome, more precisely that the recombination rate $r$ is very small $r \ll 1$, so that we consider at most one recombination, occurring on one of the two focal lineages. We distinguish cases where there is no recombination between $t=0$ and $t=T^{(f)}$, cases where the allele at the linked locus recombines (somewhere between $t=0$ and $t=T^{(f)}$ ) onto a haplotype carrying the ancestral allele at the focal locus, and cases where the allele at the linked locus recombines onto a haplotype carrying the derived allele at the focal locus. We call the second and third case homozygous and heterozygous recombination respectively, referring to the zygosity at the focal locus of the recombining pair of haplotypes (note that are three haplotypes, the two first ones have a coalescence time $T^{(f)}$, and the third one recombines with one of these two). If there is no recombination, then the coalescence time is the same for both loci, $T^{(l)}=T^{(f)}$. To treat the case with a homozygous recombination, it is convenient to name the haplotypes: $i$ and $j$ coalesce at $T_{i j}^{(f)}=T^{(f)}$ at the focal locus, and $k$ is a third haplotype, onto which the linked allele recombines 
579 (coming from $i$ ). The linked allele carried by $j$ stays on the same haplotype (no more than one recombination), and after recombining onto $k$, the linked allele initially carried by $i$ also stays on

$581 k$ (again, at most one recombination). This implies that those two linked alleles coalesce at $T_{i j}{ }^{\left({ }^{\prime \prime}\right)}=$

$582 T_{j k}(\mathrm{f})$. This time is in general different than $T_{i j}\left({ }^{(\mathrm{f})}\right.$, however on average $T_{j k}{ }^{(\mathrm{f})}$ tand $T_{i j}(\mathrm{f})$ are equal

583 (averaging over all possible coalescence trees at the focal locus). This implies that we can treat

584 the case with homozygous recombination as if there was no recombination. If there is a

585 heterozygous recombination between $i$ and $k$, at some generation between $t=0$ and $t=T^{(\mathrm{f})}$,

586 then the linked alleles still have not coalesced at $t=t_{m}$ because after the recombination one of

587 them is linked to a derived focal allele and the other one to an ancestral focal allele (and they

588 stay linked because there is at most one recombination). In that case, $T_{i j}{ }^{(1)}$ is equal to $t_{m}$ plus a

589 random time given by (on average) $T_{m}$, and is independent of $T_{i j}^{(f)}$. Using again Bayes theorem

590 and the previous results to write

$$
\begin{aligned}
\mathrm{P}\left(T^{(l)} \mid T^{(f)}\right) & =\mathrm{P}\left(T^{(l)} \mid T^{(f)}, \text { one het. rec. in }\left[0, T^{(f)}\right]\right) P\left(\text { one het. rec. in }\left[0, T^{(f)}\right]\right) \\
& +\mathrm{P}\left(T^{(l)} \mid T^{(f)}, \text { no het. rec. in }\left[0, T^{(f)}\right]\right) P\left(\text { no het. rec. in }\left[0, T^{(f)}\right]\right) \\
& =f_{m}\left(T^{(l)}-t_{m}\right)\left[1-P\left(\text { no het. rec. in }\left[0, T^{(f)}\right]\right)\right] \\
& +\delta\left(T^{(l)}-T^{(f)}\right) P\left(\text { no het. rec. in }\left[0, T^{(f)}\right]\right)
\end{aligned}
$$

595 Where $\delta(\cdot)$ is the Dirac delta function, and $f_{m}$ is the unconditional coalescence distribution of a 596 pair of lineages sampled at $t=t_{m}$, i.e. it is equal to the function $f_{0}$ introduced above but

597 replacing $t_{c}$ by $t_{c}-t_{m}$ (note also that $f_{m}(t)=0$ if $t<0$ ). We then have to evaluate the

598 probability that there is no heterozygous recombination. At generation $t$ (counted backward)

599 the probability that a linked allele recombines onto a haplotype carrying the ancestral allele at

600 the focal locus is $r\left(1-x_{t}\right)$, where $x_{t}$ is the frequency of the derived allele at the focal locus,

601 we deduce that the probability that there is no heterozygous recombination on either lineage is

602

$$
\begin{aligned}
P\left(\text { no het. rec. in }\left[0, T^{(f)}\right]\right) & =\prod_{t=1}^{T^{(f)}}\left(1-r\left[1-x_{t}\right]\right)^{2} \\
& \simeq \exp \left(-2 r \sum_{\mathrm{t}=1}^{T^{(f)}}\left(1-x_{t}\right)\right)
\end{aligned}
$$


603 This probability depends explicitly on the allele trajectory, which means that rigorously, all the

604 calculations should be conditioned on a given trajectory, and then averaged over all

605 trajectories. To allow for mathematical tractability, and to avoid heavy expressions, we consider

606 that as a good approximation $x_{t}=\bar{x}_{t}$. Finally we obtain

608

$$
\begin{aligned}
\mathrm{P}\left(T^{(l)}\right) & =E\left[\delta\left(T^{(l)}-T^{(f)}\right) \exp \left(-2 r \sum_{\mathrm{t}=1}^{T^{(f)}}\left(1-x_{t}\right)\right)\right] \\
& +f_{m}\left(T^{(l)}-t_{m}\right) E\left[1-\exp \left(-2 r \sum_{\mathrm{t}=1}^{T^{(f)}}\left(1-x_{t}\right)\right)\right]
\end{aligned}
$$

609 The expectation corresponding to this distribution yields eq. (2). generations ago

Thanks to Bayes theorem we can write

$$
\mathrm{E}\left[T^{(l)}\right]=E\left[T^{(l)} \mid T^{(l)}<t_{\mathrm{fix}}\right] P\left(T^{(l)}<t_{\mathrm{fix}}\right)+E\left[T^{(l)} \mid T^{(l)}>t_{\mathrm{fix}}\right] P\left(T^{(l)}>t_{\mathrm{fix}}\right)
$$

615 i.e. we distinguish coalescence events happening in less than $t_{\mathrm{fix}}$ generations or more than $t_{\mathrm{fix}}$

616 generations. In the former case, the coalescence is neutral, unconditional (the fixation is

617 completed) and happens in a population of constant size $N_{\mathrm{c}}$ which means that

$618 E\left[T^{(l)} \mid T^{(l)}<t_{\mathrm{fix}}\right]$ and $P\left(T^{(l)}<t_{\mathrm{fix}}\right)$ can be worked out from the neutral exponential

619 distribution. On the other hand, $E\left[T^{(l)} \mid T^{(l)}>t_{\mathrm{fix}}\right]$ is equal to $t_{\mathrm{fix}}$ plus the expectation from eq.

620 (5) which we note here $\mathrm{E}\left[T^{(l)}\right]\left(t=t_{\mathrm{fix}}\right)$. We obtain

$$
E\left[T^{(l)}\right]=2 N_{c}\left(1-e^{-t_{\mathrm{fix}} / 2 N_{c}}\right)+\mathrm{E}\left[T^{(l)}\right]\left(t=t_{\mathrm{fix}}\right) e^{-t_{\mathrm{fix}} / 2 N_{c}}
$$

622 We see that the sweep signal vanishes exponentially with the time elapsed since fixation. 


\section{Acknowledgment}

624 This work was partially supported by a Swiss NSF grant No 310030_188883 to LE. We are

625 grateful to Montgomery Slatkin, Brian Charlesworth and Jeff Jensen for their helpful comments.

626 Bibliography

627

628 Andolfatto P., and M. Przeworski, 2000 A genome-wide departure from the standard neutral model in natural populations of Drosophila. Genetics 156: 257-268.

Austerlitz F., B. Jung-Muller, B. Godelle, and P.-H. Gouyon, 1997 Evolution of coalescence times, genetic diversity and structure during colonization. Theor. Popul. Biol. 51: 148-

Barton N. H., 1995 Linkage and the limits to natural selection. Genetics 140: 821-841.

Charlesworth B., M. T. Morgan, and D. Charlesworth, 1993 The effect of deleterious mutations on neutral molecular variation. Genetics 134: 1289-1303. variation under the background selection model. Genetics 141: 1619-1632. invitational lecture. J. Hered. 104: 161-171. of Neutral Diversity? Genetics 216: 1217-1238. 
642 Corbett-Detig R. B., D. L. Hartl, and T. B. Sackton, 2015 Natural selection constrains neutral diversity across a wide range of species. PLoS Biol. 13: e1002112.

644 Crisci J. L., Y.-P. Poh, S. Mahajan, and J. D. Jensen, 2013 The impact of equilibrium assumptions on tests of selection. Front. Genet. 4: 235.

Edmonds C. A., A. S. Lillie, and L. Luca Cavalli-Sforza, 2004 Mutations arising in the wave front of an expanding population. Proc. Natl. Acad. Sci. U. S. A. 101: 975-979.

Ewens W. J., 2004 Mathematical Population Genetics: I. Theoretical Introduction. Springer, New York, NY.

Ewing G. B., and J. D. Jensen, 2016 The consequences of not accounting for background selection in demographic inference. Mol. Ecol. 25: 135-141.

Excofffier L., N. Marchi, D. A. Marques, R. Matthey-Doret, A. Gouy, et al., 2021 fastsimcoal2: demographic inference under complex evolutionary scenarios. Bioinformatics.

Excoffier L., M. Foll, and R. J. Petit, 2009 Genetic Consequences of Range Expansions. Annu. Rev. Ecol. Evol. Syst. 40: 481-501. $559-572$. 
662 Hallatschek O., and D. R. Nelson, 2008 Gene surfing in expanding populations. Theor. Popul. Biol. 73: 158-170. between selective sweeps and demography using DNA polymorphism data. Genetics

Jensen J. D., B. A. Payseur, W. Stephan, C. F. Aquadro, M. Lynch, et al., 2019 The importance of the Neutral Theory in 1968 and 50 years on: A response to Kern and Hahn 2018. Evolution 73: 111-114.

Johri P., B. Charlesworth, and J. D. Jensen, 2020 Toward an Evolutionarily Appropriate Null Model: Jointly Inferring Demography and Purifying Selection. Genetics 215: 173-192.

672 Johri P., B. Charlesworth, E. K. Howell, M. Lynch, and J. D. Jensen, 2021a Revisiting the 673 Notion of Deleterious Sweeps. Genetics. https://doi.org/10.1093/genetics/iyab094

674 Johri P., K. Riall, H. Becher, L. Excoffier, B. Charlesworth, et al., 2021b The Impact of Purifying and Background Selection on the Inference of Population History: Problems

Kaiser V. B., and B. Charlesworth, 2009 The effects of deleterious mutations on evolution in and Prospects. Mol. Biol. Evol. 38: 2986-3003. Genetics 123: 887-899.

681 Kingman J. F. C., 1982a The coalescent. Stochastic Process. Appl. 13: 235-248. 
682 Kingman J. F. C., 1982b On the genealogy of large populations. J. Appl. Probab. 19A: 27-43.

683 Klopfstein S., M. Currat, and L. Excoffier, 2006 The fate of mutations surfing on the wave of a range expansion. Mol. Biol. Evol. 23: 482-490.

Maruyama T., and M. Kimura, 1975 Moments for sum of an arbitrary function of gene frequency along a stochastic path of gene frequency change. Proc. Natl. Acad. Sci. U. S. A. 72: $1602-1604$.

Mathew L. A., and J. D. Jensen, 2015 Evaluating the ability of the pairwise joint site frequency spectrum to co-estimate selection and demography. Front. Genet. 6: 268.

Maynard Smith J., and J. Haigh, 1974 The hitch-hiking effect of a favorable gene. Genet. Res. 23: $23-35$.

692 Nicolaisen L. E., and M. M. Desai, 2013 Distortions in genealogies due to purifying selection and recombination. Genetics 195: 221-230.

694 O’Fallon B. D., J. Seger, and F. R. Adler, 2010 A continuous-state coalescent and the impact of weak selection on the structure of gene genealogies. Mol. Biol. Evol. 27: 1162-1172.

696 Orozco-terWengel P., M. Kapun, V. Nolte, R. Kofler, T. Flatt, et al., 2012 Adaptation of Drosophila to a novel laboratory environment reveals temporally heterogeneous trajectories of selected alleles. Mol. Ecol. 21: 4931-4941. deleterious mutations during range expansions. Mol. Ecol. 22: 5972-5982. 
701 Peischl S., and L. Excoffier, 2015 Expansion load: recessive mutations and the role of standing 702 genetic variation. Mol. Ecol. 24: 2084-2094.

Pouyet F., S. Aeschbacher, A. Thiéry, and L. Excoffier, 2018 Background selection and biased gene conversion affect more than $95 \%$ of the human genome and bias demographic inferences. Elife 7: e36317.

Pouyet F., and K. J. Gilbert, 2019 Towards an improved understanding of molecular evolution: the relative roles of selection, drift, and everything in between. arXiv [q-bio.PE]. gene Quetzalcoatl in Drosophila melanogaster. Proc. Natl. Acad. Sci. U. S. A. 107:

711 Rousselle M., M. Mollion, B. Nabholz, T. Bataillon, and N. Galtier, 2018 Overestimation of the adaptive substitution rate in fluctuating populations. Biol. Lett. 14. https://doi.org/10.1098/rsbl.2018.0055

714 Schrider D. R., and A. D. Kern, 2018 Supervised Machine Learning for Population Genetics: A New Paradigm. Trends Genet. 34: 301-312.

716 Slatkin M., 1996 Gene genealogies within mutant allelic classes. Genetics 143: 579-587.

717 Sousa V., S. Peischl, and L. Excoffier, 2014 Impact of range expansions on current human 718 genomic diversity. Curr. Opin. Genet. Dev. 29: 22-30.

719 Tajima F., 1990 Relationship between DNA polymorphism and fixation time. Genetics 125: $447-454$. 
721 Tavaré S., 1984 Line-of-descent and genealogical processes, and their applications in population genetics models. Theor. Popul. Biol. 26: 119-164.

723 Teshima K. M., G. Coop, and M. Przeworski, 2006 How reliable are empirical genomic scans for $724 \quad$ selective sweeps? Genome Res. 16: 702-712.

725 Thornton K. R., and J. D. Jensen, 2007 Controlling the false-positive rate in multilocus genome scans for selection. Genetics 175: 737-750.

727 Wares J. P., 2009 Evolutionary dynamics of transferrin in Notropis. J. Fish Biol. 74: 1056-1069.

728 Weissman D. B., and N. H. Barton, 2012 Limits to the rate of adaptive substitution in sexual populations. PLoS Genet. 8: e1002740.

730 Zhao L., M. Lascoux, A. D. J. Overall, and D. Waxman, 2013 The characteristic trajectory of a 731 fixing allele: a consequence of fictitious selection that arises from conditioning. Genetics 195: 993-1006. 